\title{
Commentary
}

\section{The MOGE(S) Classification of Cardiomyopathy}

\author{
Eloisa Arbustini ${ }^{1}$, Jagat Narula ${ }^{2}$, Nidhi Priya ${ }^{3}$, Sadik Raja Panwar $^{4}$ \\ ${ }^{1}$ Institute of Inheritable Diseases, Pavia, Italy; ${ }^{2}$ Icahn School of Medicine at Mount Sinai, \\ New York, NY, USA; ${ }^{3}$ Assistant Professor, Department of Pathology, RUHS College of \\ Medical Sciences, Jaipur; ${ }^{4}$ Consultant, Interventional Cardiologist, Beckley ARH Hospital, \\ Beckley, West Virgina, USA.
}

The "M" descriptor carries a subscript entry based on the morpho-functional phenotype represented by the initial letter of each type or subtype of cardiomyopathy. $\mathrm{H}$ stands for Hypertrophic Cardiomyopathy, D for Dilated Cardiomyopathy; R for Restrictive Cardiomyopathy and R EMF for Restrictive Endomyocardial Fibrosis, ACM for Arrhythmogenic Cardiomyopathy, LVNC for Left Ventricular Noncompaction. As an important notation, E and 0 (zero) were introduced. E stands for early diagnosis of cardiomyopathy and applies to patients demonstrating familial/genetic markers of disease but not yet phenotypically fulfilling the diagnostic criteria of the cardiomyopathy; 0 explains the yet unaffected status in genetically affected sibling or off spring. Notation "NS" gives the possibility of describing a non-specific (nonclassifiable) phenotype such as the segmental hypokinesis of the left ventricle, single papillary muscle hypertrophy, etc. that may not conform with the diagnostic criteria of the most common types of cardiomyopathies. The "NA" notation is applied when diagnostic information is not available or relatives deceased. Although poorly informative, even NA can be useful in family studies especially when reporting on obligate or non obligate carriers of mutations identified in the family and segregating or non segregating with the phenotype on the basis of the cascade family screening.

The "O" stands for Organ involvement, which in addition to the heart may involve the skeletal muscle, nervous system, liver, kidney, ocular, auditory, cutaneous systems, lung, etc. The first letter of the organ involved should be subscripted. This allows easy identification of syndromic patterns.

The descriptor " G" is for Genetics to describe if the disease is heritable and in this case, the mode of transmission of disease is explained in the subscript such as $\mathrm{AD}$ for autosomal dominant, and $\mathrm{AR}$ for autosomal recessive. All these considerations are part of the medical process of family evaluation.

The $\mathbf{E}$ (etiology) is the most important descriptor because it allows the description of disease genes and mutations in genetic diseases as well as the description of an infectious, toxic or degenerative basis in non-genetic variety of cardiomyopathy. It is described as a dual subscript; the first subscript defines if it is genetic or nongenetic. The second subscript explicitly defines the genetic and nongenetic disease etiology. When $\mathbf{E}$ (Table 1) describes the genetic origin, the gene mutation if available should be described. If the disease is familial, but genetic cause is not identified or not sought, then it must be stated as such.

The notation " $\mathrm{S}$ " is optional. In two parts, it represents the ACC/AHA stage of the disease and in second part the symptomatic stage is offered through the New York Heart Association (NYHA) classification.

For an easy introduction of the MOGE(S) classification, an app has been developed. The use of the app facilitates the description of each single cardiomyopathy in a hierarchical process where the first description is the morphofunctional phenotype (M), followed by specification of the organ $(\mathrm{O})$ involvement (heart only or other organs and tissues), whether the disorders is familial/genetic (G) or non familial or unknown, unproven and, in case of familial diseases, the addition of the mode of inheritance, based on clinical pedigree and screening of the family, and finally the etiology (E) genetic or non genetic. 
Table 1 : The MOGE(S) Nosology System for classifying cardiomyopathy patients ${ }^{1,2}$

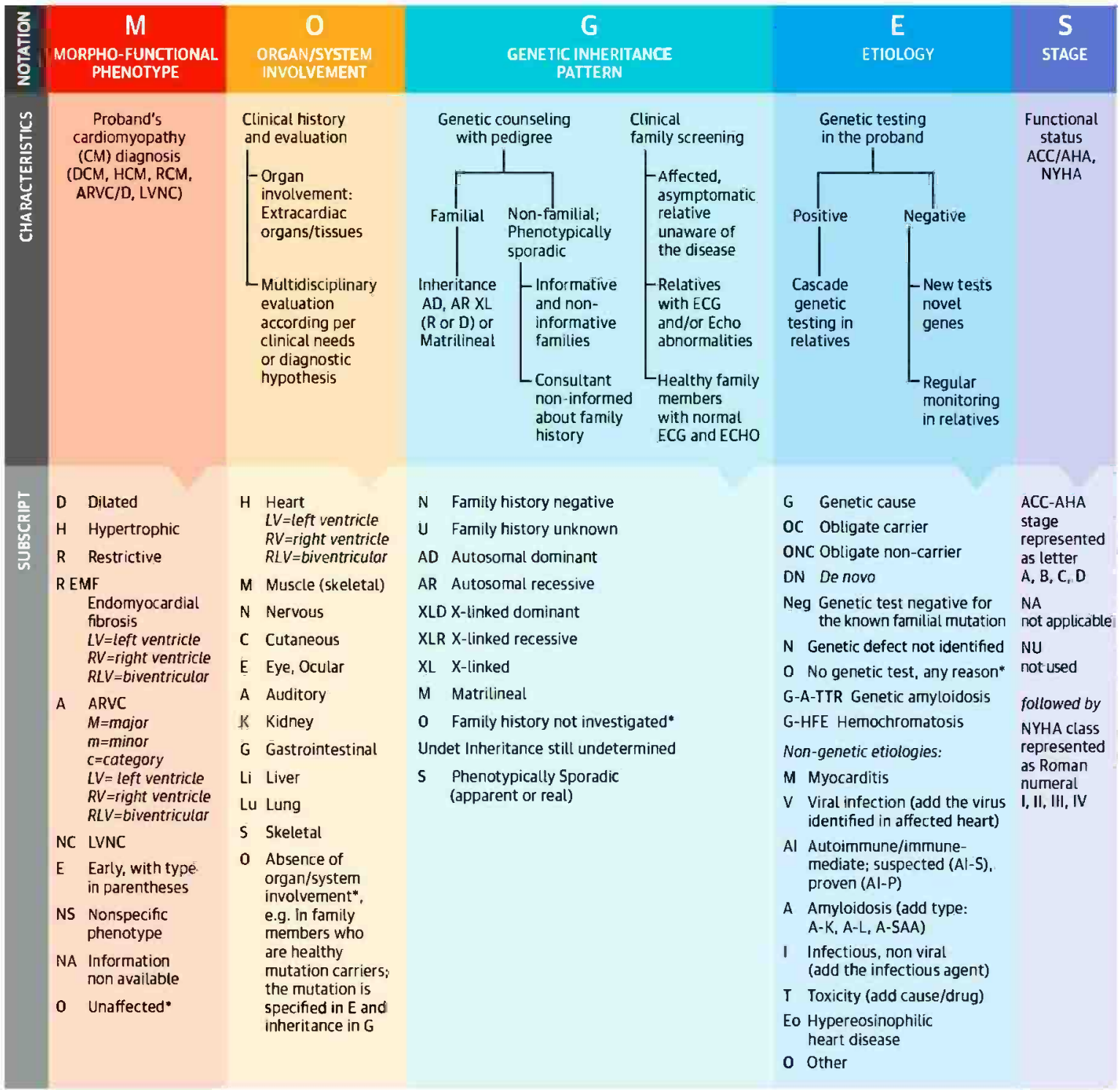

Evaluation of cardiomyopathy patients and development of MOGE(S) nosology. (M) The morphofunctional phenotype description may contain more information using standard abbreviations: AVB = atrioventricular block; LQT = prolongation of the QT interval; $\downarrow \mathrm{PR}=$ short PR interval; $\downarrow \mathrm{R}=$ low electrocardiographic voltages; WPW = Wolf Parkinson White syndrome; and other clinical red flags. These red flags are to be placed in parentheses after the notation of morphofunctional phenotype. Overlapping (H+R), (D+A), $(\mathrm{NC}+\mathrm{H}),(\mathrm{H}+\mathrm{D}),(\mathrm{D}+\mathrm{NC})$ or more complex combinations such as $(\mathrm{H}+\mathrm{R}+\mathrm{NC}) .{ }^{*}$ Notation is zero $(0)$ not the letter "O." (E) The etiologic annotation provides the description of the specific disease gene and mutation, as well as a description of nongenetic etiology. Even when genetic analysis is not available, the (G) may inform about a genetic disease, supporting family monitoring strategies. \#According to the Human Genome Variation Society, genetic variants should be classified based on their effects on gene function as: affecting function, probably affecting function, unknown (variants of unknown significance [VUS]), probably not affecting function, and not affecting function. A color code assigned to each variant can provide information about the potential role of the identified variant: affects function or probably affects function (red); Variant of Unknown Significance (VUS) (yellow); and probably does not affect function (or probably no functional effect) or does not affect function (no functional effect) (green). The compilation is guided by the MOGES app. $\mathrm{ACC}=$ American College of Cardiology; $\mathrm{AHA}=$ American Heart Association; $\mathrm{ARVC} / \mathrm{D}=$ arrhythmogenic right ventricular cardiomyopathy/dysplasia; $\mathrm{DCM}=$ dilated cardiomyopathy; $\mathrm{ECG}=$ electrocardiogram; $\mathrm{ECHO}=$ echocardiogram; HCM = hypertrophic cardiomyopathy; LVNC = left ventricular noncompaction; NYHA = New York Heart Association; $\mathrm{RCM}=$ restrictive cardiomyopathy. 
In case of genetic cardiomyopathy, when the mutation is identified or when more than one mutation/genetic variant is identified, the color code describes possibly and probably pathologic mutations (RED), genetic variants of unknown significance (YELLOW) and single nucleotide polymorphism (SNPs) with possible functional significance (GREEN).

\section{CONCLUSION}

In this transition era between the descriptive, phenotypically based, and etiology-based molecular medicine, the past cannot be abandoned as it provides the strength of all what we know, but the future cannot be ignored. While maintaining the descriptive phenotypebased diagnosis as the leading item in the hierarchical nosology system, MOGE(S) also provides information on causes/etiology and obligates cardiologists to a step-bystep clinical work-up in which the probands (as a whole person and not as a heart) his/her family, the etiologic clues are all considered and described.

\section{REFERENCES:}

1. Arbustini E, Narula N, Dec GW, Reddy KS, Greenberg B, Kushwaha S, Marwick T, Pinney S, Bellazzi R, Favalli V, Kramer C, Roberts R, Zoghbi WA, Bonow R, Tavazzi L, Fuster V, Narula J.The MOGE(S) classification for a phenotype-genotype nomenclature of cardiomyopathy: endorsed by the World Heart Federation. J Am Coll Cardiol. 2013; 62:2046-72. Erratum in: J Am Coll Cardiol. 2014;63:191-4.

2. Arbustini E, Narula N, Dec GW, Reddy KS, Greenberg B, Kushwaha S, Marwick T, Pinney S, Bellazzi R, Favalli V, Kramer C, Roberts R, Zoghbi WA, Bonow R, Tavazzi L, Fuster V, Narula J. Reply: The MOGE(S) Classification for a Phenotype-Genotype Nomenclature of Cardiomyopathy: More Questions Than Answers? J Am Coll Cardiol. 2014; 63:2584-6.

\section{Corresponding Author:}

Dr. Jagat Narula, Icahn School of Medicine at Mount Sinai, New York, NY, USA. 